\title{
Phoenix: Fault-Tolerant Distributed Web Storage based on URLs
}

\author{
J. L. Gonzalez* ${ }^{1}$, Ricardo Marcelín-Jiménez ${ }^{2}$ \\ ${ }^{1}$ Dept. Systems and Computation \\ Cd. Valles Institute of Technology \\ Carretera al Ingenio Km2, Cd. Valles, S.L.P. Mexico \\ ${ }^{2}$ Electrical Eng. Dept. \\ UAM-Iztapalapa \\ Atlixco 186, 09340 Iztapalapa, Mexico City \\ joseluig@ac.upc.edu calu@xanum.uam.mx
}

\begin{abstract}
This paper presents, in the form of a case study, the design, implementation and performance evaluation of Phoenix: a prototype of a fault-tolerant distributed storage web service based on URLs. Our system supports a set of redundancy strategies including simple file-replication, error-coding techniques and a hybrid adaptive technique. The web storage middleware (WSM), which is at the core of the prototype, reuses the URLs of the files to dynamically accommodate data and redundancy load.
\end{abstract}

Experimental assessments show that distributing the users to several web servers compensates for the overhead produced both by generating and distributing data redundancy. We also found that during a non-critical number of web server failures, users do not perceive but a lengthening on the system response times. As expected, peak user requests and network congestion are the most important factors affecting the storage system's performance.

Keywords; fault-tolerance; redundancy; web; storage; virtualisation;

\section{INTRODUCTION}

The Internet has fostered the development of new web tools, including data-sharing and on-line collaborative work applications. Information availability and integrity have become critical issues in these new environments because of the dramatic increase in the number of Internet users and its direct impact on data production [1,2].

Web storage services seem to be a feasible solution targeted at providing individuals with an effective backup.

A typical user may recover information when his/her notebook has crashed or while he/she is out of the office and requires access to a given set of files which is not at hand. Nevertheless, web storage as monolithic solution means one single failure point reducing the reliability of the whole system. In addition, peak user requests and network congestion could affect the performance of storage systems.

These conditions deserve more attention from web system designers with a special focus on distributed fault-tolerant storage systems.

A distributed storage system is a collection of

This research was partially supported by "El Centro Público de interconnected storage devices that contribute with their individual capacities to create an extended system offering improved performance. Although the simplest function of such a system is to spread a collection of files across the storage devices attached to a network, the desirable attributes of quality must also be incorporated [3,4].

In many storage scenarios, there is a file to be stored on a hard disk belonging to a network component. Nevertheless, if this component goes out of service the information retrieval would be cancelled. A common approach found in this condition is the utilisation of a redundancy strategy in order to provide fault-tolerance and, particularly, integrity. In this context, the simplest solution consists of file replication $[5,6]$.

A file to be stored can be split into various information units or kept as a single one. In any case, several copies of the resulting units are allocated to a given subset of storage devices. In contrast, there systems exist where information redundancy is implemented using error correcting codes [7,8]. In this approach, a file is divided into fragments. Subsequently, each fragment is transformed using a particular error-coding technique (e.g. Reed-Solomon, IDA, network codes, fountain codes, etc.). In the last step, the coded fragments are copied and again allocated to a given subset of the available storage devices.

The decision regarding the strategy that provides information redundancy has a great impact on operational aspects such as cost and management [9].

Replication offers a very simple retrieval mechanism, but it may require an excessive cost with respect to information storage.

In contrast, error-coding can provide similar levels of availability, using a very limited amount of storage. Nevertheless, tracking all the pieces that make up each and every file may become a tremendous challenge.

Recent studies [10] suggest that when devices offer a longterm stable service, systems might profit from conservative error-coding. On the other hand, those systems where devices offer an intermittent service should be built on the basis of aggressive replication. Intermediate solutions, with combined replication and coding techniques, are also suggested in order to facilitate information retrieval and tracking.

Información y Documentación para la Industria (INFOTEC).” 
The virtualisation of a distributed fault-tolerant storage system as a web storage solution has become a common solution in order to provide both data availability and reliability in web applications. This is the case of storage services such as Amazon S3, Nirvanix CloudNAS, or Microsoft SkyDrive, which have become successful businesses offering permanent file availability.

However, the following concerns arise with these kinds of service:

- $\quad$ The price depends on the amount of information and the time this information remains in the cloud. Trends show that in the middle-term it is foreseen an exponential growth on the amount of information trusted to external storage providers, which means that service prices will be affected [11].

- $\quad$ Users ignore the actual place where their information is located. This could be an important issue that threatens business continuity and information confidentiality.

Over the last few years the cost of external web storage has been observed to be several times higher than the value of the disks involved [12]. In order to face up to this kind of issues, in-house web storage offers the possibility of building a costeffective and quality service, based on commodity components.

This paper presents, in the form of a case study, a prototype called Phoenix, which is a fault-tolerant storage system based on a set of distributed low-cost web servers.

Web applications work with URL's to handle user's contents. Our system is developed as a web storage middleware (WSM) that supports file management by re-using the same URL information. As a result, sharing content among servers is an immediate possibility that can be easily accomplished. The system uses the capacity of all web servers as one single virtualised partition by using the WSM.

Phoenix is able to withstand a given number of crash failures without interrupting its service due to an effective allocation middleware in combination with a set of redundancy strategies, such as replication and error-coding.

Our first experiments show that distributing the users over several web servers compensates for the overhead involved in redundancy management. In addition, metadata can be reused to build efficient, private and fault-tolerant distributed web storage. This proposal becomes an interesting alternative to external web storage providers because it offers a cost-effective, fault-tolerant, in-house storage solution.

\section{RELATED WORK}

Online storage applications such as Amazon S3, Nirvanix CloudNAS, or Microsoft SkyDrive are becoming a common service available on the Internet. Currently, this kind of application represents a business success because they offer solutions to those users asking for permanent file availability.

Web storage services seem to be a feasible solution targeted at providing individuals with an effective backup. A typical user may recover information when his/her notebook crashes or while he/she is out of the office and requires access to a given set of files which is not on hand.

Service providers use their private capacities and redundancy strategies according to their own concerns. Some of these applications offer free storage services but with performance penalisation [13], others apply membership approaches [14], while the most popular (Amazon S3) applies rates based on monthly stored content [15].

Reliability in cloud storage is still an open issue that has started to be addressed by schemes such as secure RAID for the Cloud [16] and P+Q [17] using VLSD [18]. This means that organisations requiring intensive storage may not be the intended clients of current providers. In this context, in-house on-line storage is an interesting solution and the object of our proposal. Studies exist that show storage schemes based on IDA for LAN [19].

\section{PHOENIX DESIGN PRINCIPLES}

In Phoenix, the storage components, also called WS-Nodes, are organised as a redundant set of web servers, called Web Storage Cell or WS-Cell.

Each WS-Cell is coordinated by one WS-Node, also called gateway, which is charged to provide the entry point to the system.

WS-Nodes are regarded as part of a logic ring, i.e. their identities are ordered according to a cyclic list. When a user contacts the gateway and logs in, they are immediately transferred to the first idle WS-Node in this list. The WS-Node in charge displays a web page showing their files and profile and supports the user's request.

Each new user account produces a separate folder in the file system of each WS-Node. This procedure yields a unified structure available at any point of the WS-Cell. As a result, WSM virtualises the sum of the WS-Node's capacities to offer a single partition or single space address.

Web applications use metadata based on URLs to handle user content. A URL is a path including the domain/IP of the web server, the path of the profile folder and the name of a given file. The URLs are recorded onto the web page database and they are used to handle the files in the user folders.

In turn, redundancy strategies also involve data placement and metadata management. Therefore, an extended metadata operation is required to support web storage. This means that the work-flows may require more search points and it might be very difficult to enforce a centralised control on the corresponding storage platform.

In order to affect as little as possible the regular behaviour of the web pages, Phoenix reuses the URLs of the same page to control file accommodation and recovery within the WS-Cell and uses them in a deterministic placement method for handling data and redundancy. As a side-effect it also reduces the complexity of the storage operations.

\section{A. WSM: A Web Storage Middleware}

Phoenix provides the users with two basic operations: files upload and download. We thus define a WSM which is located between the web applications and the file system. The main function of this WSM is the interception and execution of users' requests in a transparent manner. The WSM has been designed as a stack of layers that can be included in web pages. These layers include the following modules:

1. Data Indirection Module: This module offers location transparency when downloading or uploading files in the WSCell. It reuses the URLs obtained from the web page to determine the final course of a user's request. In Phoenix the 
upload operations are always performed in a local manner. By local we mean that the uploaded file is stored at the WS-Node in charge. As a result, the files of a single user could be located at different WS-Nodes.

In contrast, download operations could be performed in three different ways:

- As a local operation, when the WS-Node in charge is able to retrieve the file for the user by itself. only extra metadata added by the middleware. This table is used when downloading data and distributing redundancy within the WS-Cell. This table is also used when a gateway node is unavailable. In this case, a polling event among the rest of the WS-node performed in the background determines the new gateway by following the sequence of WS-nodes table. This means WS-node1 is the first candidate when the gateway dies and WS-node2 is the next one, and so on.

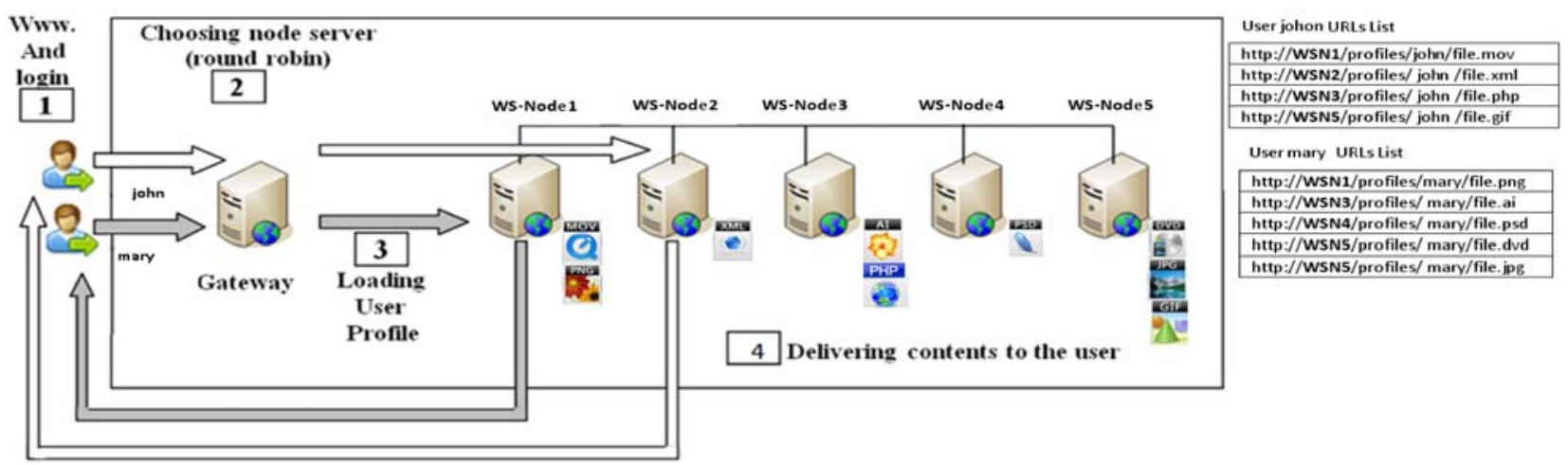

Figure 1. An example of a WS-Cell in Phoenix.

- $\quad$ As an on demand service, when the file has not been stored at the WS-Node in charge. In this case, the WS-Node contacts the location(s) in the WS-Cell where the file has actually been located and recovers it. Afterwards, it is able to give the file back to the user.

- As a transfer, when the size of the requested file exceeds a maximum (\%5MB). In this case, the user is retransferred to the server where the file is actually located.

Fig. 1 shows the two main functions supported by the system in an example. In this figure, user Mary (step 1) is uploading a file and WS-Node1 will be charged to complete her requests (step 2) by following the URLs list of this user (steps 3 and 4). In the same figure, user John is downloading a previously stored file. Now, WS-Node2 is in charge to retrieve it. As we can see, the users are distributed according to a round robin service policy.

2. Redundancy Module: This module supports failure transparency based on 2 actions, proactive and reactive:

- $\quad$ It produces redundant information for a new uploaded file about to be accommodated, according to the redundancy strategy assigned to the user's account (more details in a further section).

- It gathers redundant information in order to rebuild a file, when the server where it was initially located is currently unavailable. When the system operates in this condition is considered to be degraded.

3. Distributor Module: This module supports information transport among the nodes of the WS-Cell. It depends on the redundancy strategy to allocate information or retrieve it from a given subset of repositories. This module can be configured with the distribution rules of a given redundancy scheme and it is applied in the WS-Cell. Fig. 2 shows the communication stack used by WSM during storage operations.

4. Metadata Management: In Phoenix the IP/domain of each WS-node is mapped onto the WS-nodes table. This is the

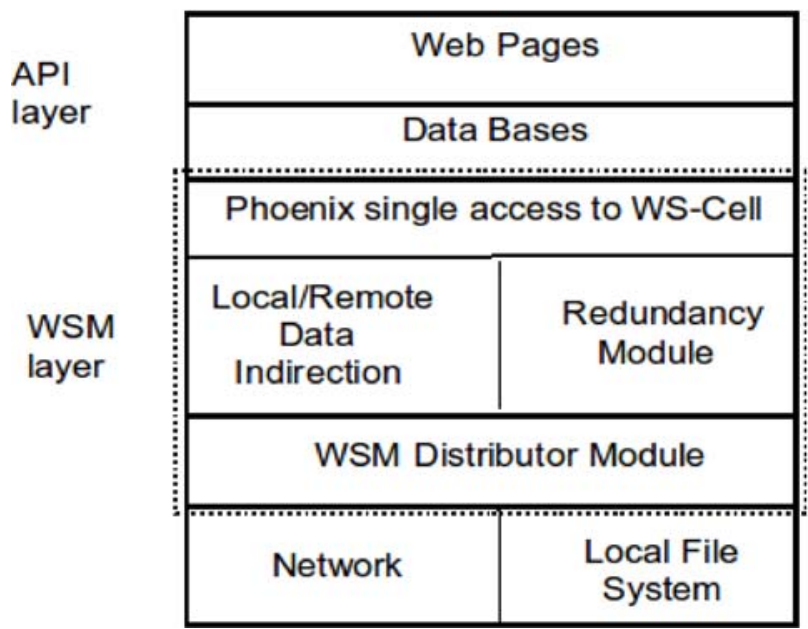

Figure 2. The communication stack used by Phoenix

\section{B. Redundancy Strategies}

The redundancy module that makes part of the WSM can be configured in order to provide different redundancy strategies. By this term we mean the redundancy generation function followed by data allocation. Two basic redundancy strategies are currently supported: simple replication based on a chained declustering strategy [20] and codification based on IDA storage schemes [21]. A hybrid approach combining both of them is also possible.

\section{Redundancy based on Simple Replicas}

The chained declustering strategy is based on simple file replication. Two replicas of a given file have to be stored. The first copy is allocated to a primary storage node, while the second is allocated to a different node, also called secondary or mirror. The two appointed components can be selected by using a scheduling function which is available to every storage participant. 
In this case, the scheduling function is implemented as a cyclic successor function on the identities of the nodes of the WS-Cell, i.e. primary and mirror are successively appointed according to a cyclic designation function.

\section{Redundancy based on IDA}

A storage scheme is a distributed coordination procedure that operates on a set of network components or locations and is executed through various steps. Every component is in charge of its own disk. Each time a step is triggered, a subset of locations (to be referred to as a block) is selected to participate in storage placement. Every component knows the roles involved in this process. Blocks are cyclically rescheduled. Storage schemes can be considered as a particular form of space redundancy [22].

This strategy proposes the use of storage schemes based on the Information Dispersal Algorithm, IDA $(k, m)$, as a means to provide information integrity (IDA is part of the Reed-Solomon family of error-correcting codes). Let $F$ be a file of size $|F|$ that is transformed into $n$ new files, called dispersals. Each dispersal is of size $|F| / m$, where $\mathrm{n}>m$. Dispersals are stored in $n$ different points that make up part of the block in charge of the current storage operation. From the algorithm properties, it is granted that the original file can be reconstructed, provided that any $\mathrm{m}$ dispersals are available.

The combination of parameters $(n, m)$ is able to support up to $(n-m)$ missing dispersals at the price of an excess of redundancy equal to $(n-m) / m$.

Table I shows, at the intersection of row $\mathrm{k}$ and column $\mathrm{m}$, the excess \% of storage capacity required to support this combination of parameters.

In Phoenix, each server handles data by using an implementation of IDA with parameters $(5,3)$. This means that each file is transformed into $n=5$ dispersals and the file will remain available provided that any $m=3$ dispersals are at hand. This implies that the system spends an excess of $66.7 \%$ on redundancy overhead.

\section{DESIGN OF EXPERIMENTS}

We built a WSM-Cell prototype using six PCs offering an overall capacity of 480 GB. (See features of PCs in Table II).

The servers were configured using Apache2. In this prototype, each server was assigned to a public IP address. All servers were connected to a Cisco Router 2800, achieving a 2 $\mathrm{Mb}$ transfer rate.

\section{A. Studied Configurations}

The following Phoenix configurations are defined to be stressed, as well as the parameters under evaluation.

- Centralised: This configuration works as a reference or control, and is based on one single web server having the same characteristics as each of the PCs used in the prototype.

- Distr-NR-NO: In this configuration, the WSM works as a distributed web system composed of five WS-nodes and one WS-gateway. NO (Normal Operation) means that during the test all WS-nodes were available and NR means that system is neither producing, nor distributing redundancy.

- Distr-CD-NO: Same as Distr-NR-NO, but this time producing and distributing simple redundancy with the Chained Declustering allocation strategy.

TABLE I. IDA PARAMETERS COMBINATION

\begin{tabular}{|c|c|c|c|c|c|c|}
\hline \multicolumn{7}{|c|}{$\mathbf{m}$ (Dispersals required for recovering files) } \\
\hline $\begin{array}{c}\mathbf{n} \\
\text { Server }\end{array}$ & $\mathbf{m}=\mathbf{1}$ & $\mathbf{m}=\mathbf{2}$ & $\mathbf{m}=\mathbf{3}$ & $\mathbf{m}=\mathbf{4}$ & $\mathbf{m}=\mathbf{5}$ & $\mathbf{m}=\mathbf{6}$ \\
\hline $\mathbf{n = 2}$ & $100 \%$ & & & & & \\
\hline $\mathbf{n}=\mathbf{3}$ & $200 \%$ & $50 \%$ & & & & \\
\hline $\mathbf{n = 4}$ & $300 \%$ & $100 \%$ & $33.3 \%$ & & & \\
\hline $\mathbf{n}=\mathbf{5}$ & $400 \%$ & $150 \%$ & $66.70 \%$ & $25 \%$ & & \\
\hline $\mathbf{n = 6}$ & $500 \%$ & $200 \%$ & $100 \%$ & $50 \%$ & $20 \%$ & \\
\hline $\mathbf{n = 7}$ & $600 \%$ & $250 \%$ & $133.3 \%$ & $75 \%$ & $40 \%$ & $16.7 \%$ \\
\hline
\end{tabular}

(at least) to support fault-tolerance

TABLE II.

FEATURES OF PROTOTYPE COMPONENTS

\begin{tabular}{|c|c|c|}
\hline \multicolumn{3}{|c|}{ Type of server } \\
\hline Characteristics & $\begin{array}{c}\text { WS-Nodes } \\
\text { HP-Desktop PCs }\end{array}$ & $\begin{array}{c}\text { Centralised } \\
\text { HP Proliant ML100 } \\
\text { Server }\end{array}$ \\
\hline Storage & $\begin{array}{l}\text { SATA } 3.0 \mathrm{~Gb} / \mathrm{s} 7200 \\
\text { RPM } 80 \mathrm{~GB}\end{array}$ & SATA 7.2K $160 \mathrm{~GB}$ \\
\hline $\mathrm{CPU}$ & $\begin{array}{l}\text { Intel Core } 2 \text { Quad } \\
\text { Intel }\end{array}$ & $\begin{array}{l}\text { Intel Core } 2 \text { Quad } \\
\text { Intel }\end{array}$ \\
\hline RAM & $\begin{array}{l}1 \text { Gb PC2-6400 } \\
\text { DDR" SDRAM(800 } \\
\text { Mhz) }\end{array}$ & $\begin{array}{l}1 \text { Gb PC2-6400 } \\
\text { DDR" SDRAM(800 } \\
\text { Mhz) }\end{array}$ \\
\hline Network Card & Fast Ethernet & Fast Ethernet \\
\hline $\begin{array}{l}\text { Operating } \\
\text { System }\end{array}$ & Ubuntu 10 & Ubuntu 10 \\
\hline
\end{tabular}

The characteristics of WS-Nodes. and the centralised server.

- $\quad$ Distr-IDA-NO: Same as Distr-NR-NO, but producing and distributing five dispersals by using IDA and the allocation method based on storage schemes.Distr-IDA-NO F(i): Same as Distr-IDA-No, but this time the system is under faulty conditions because i WS-Nodes are unavailable.Distr-CD F(i): Same as in Distr-IDA F(i) but applying a simple redundancy with a chained declustering distribution.

In all configurations we tested an application web page called Adaptive Collaborative Working (ACW) with either 12, 24 or 30 simultaneous users storing and retrieving data in a mean of 8 files of 882.91, 737.29 and 575.27 KB from two different cities in the same region. Users were only required to handle files with a minimum size of $300 \mathrm{~KB}$. Only $10 \%$ of the users required more than $5 \mathrm{MB}$ size per file during the experiments. 


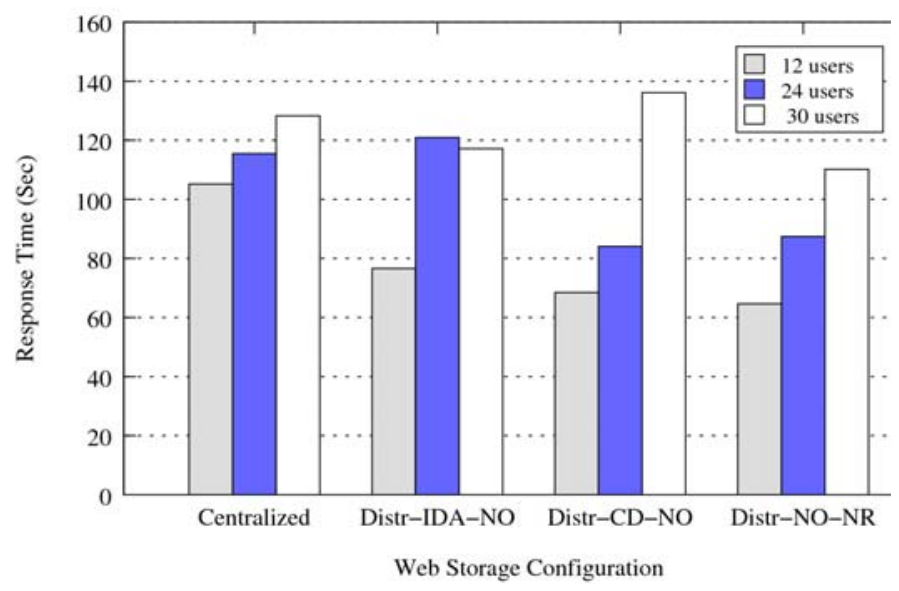

Figure 3. Mean response time of upload operations. for either 12, 24 or 30 simultaneous users.

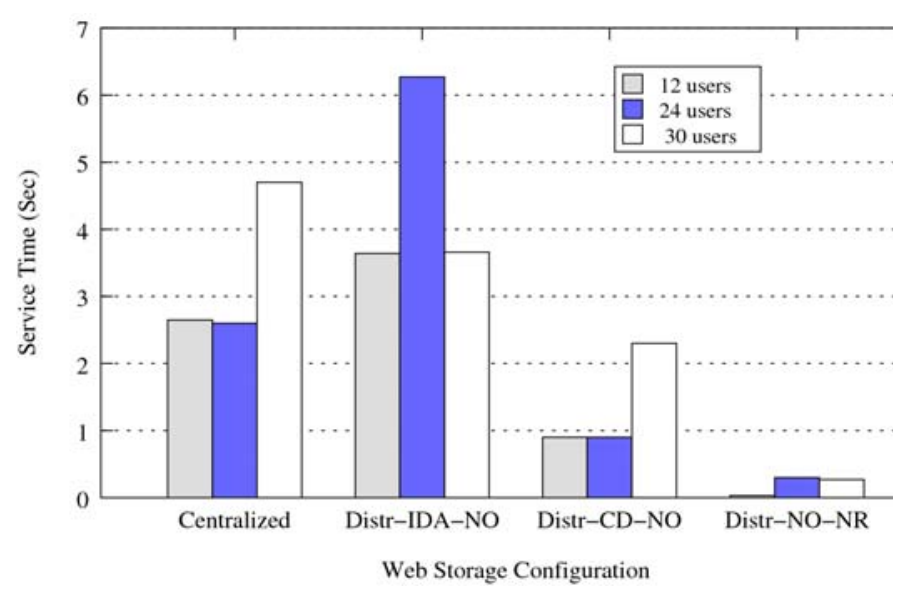

Figure 4. Mean service times of uploading files.

It can be observed that Distr-NR-NO produces the smallest response times. This result can be explained by the fact that five WS-Nodes are sharing their workload (file reception and its allocation within WS-Cell) without producing redundancy (NR) on the fly.

In contrast, Distr-IDA-NO and Distr-CD-NO require an additional processing time for producing and distributing redundancy. As a result, both configurations are less efficient than Distr-NR-NO.

When upload operations are performed on the fly, the control of the web page is only returned to the user when the system is able to recover the uploaded file from the involved WS-Nodes. In order to improve Distr-IDA-NO and Distr-CD times, redundancy production and distribution could be performed during idle moments, but this approach may create a window of vulnerability increasing the probability of data losses.

In Fig. 3 we also can see that the Centralised configuration (regular web pages handling profiles) shows the behaviour of a potential bottleneck. In fact, its response times are similar to Distr-IDA-NO.

The relative behaviour of these configurations is repeated when the number of users grows. Nevertheless, as could be expected, there is a mean performance penalisation of $5 \%$. The overhead of producing and distributing redundancy seems to be compensated for by the distribution of users among several web servers.

- $\quad$ The Effects of the Redundancy Management on Web Storage Performance: The effects of production and distribution of redundancy can be observed in detail in the service times during upload operations.

Fig. 4 also shows service times. In this figure we can see that Distr-IDA and Distr-CD both spend more time at the server side than Distr-NR-NO and Centralised because they produce and distribute redundancy. Distr-IDA-NO produces and distributes five dispersal files and thus it is the configuration with more performance restrictions. Nevertheless, comparing Fig. 3 (showing response times) and Fig. 4 (showing service times) we can observe that response times are measured in tens

Fig. 3 shows the mean response times per request, corresponding to each web storage configurations being tested, 
of seconds (even minutes) and service times in only seconds.

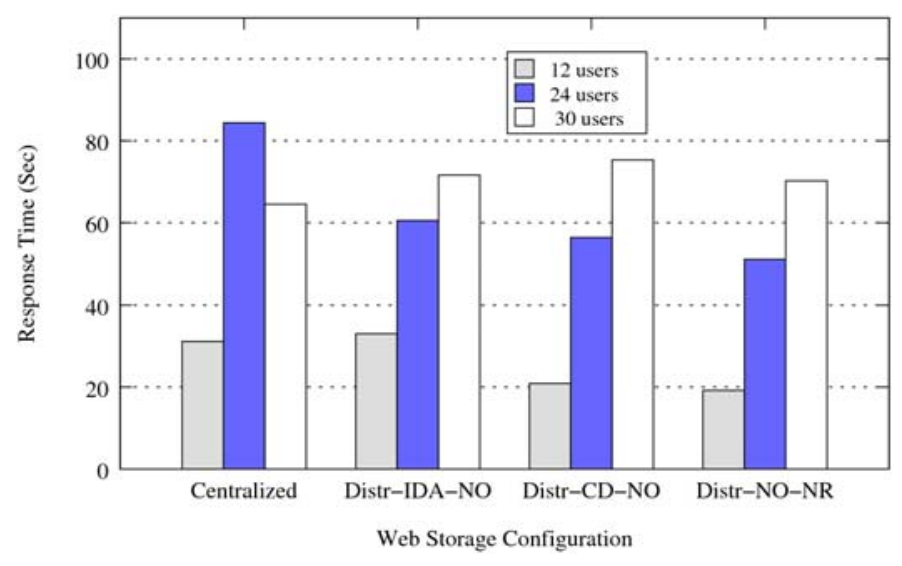

Figure 5. Mean response time of download operations.

This difference means that in all cases Internet communications produce the strongest impact on the upload operation performance.

The production and distribution redundancy only represents a fraction of the overall upload operations price, which means that fault-tolerance distributed web storage such as Phoenix is a feasible solution.

\section{B. Download Operations}

Fig. 5 shows the response times per configuration when downloading files. As we can see, the behaviour is similar to the upload operations but the service times are reduced in all cases. We surmise that this can be explained because of the fact that ISP providers generally offer asymmetrical links where downstream rates are better than upstream.

In this graph we can also see that Distr-IDA-NO produces similar response times to those from Distr-NR-NO because both systems provide an on demand service.

- $\quad$ The Effects of the On-demand Service on Web Storage Performance: An optimal scenario appears when a user is dispatched by the WS-Node where its file has been stored because WSM obtains the file from the local file system.

Otherwise, this file is obtained from its original WS-Node by using the on demand service. This could result in costs of network transportation, some computation and metadata management, which can be observed on the service times during download operations.

Fig. 6 shows the service times per configuration when downloading files. As we can see, the service times for distributed configurations Distr-NR-NO, Distr-IDA-NO and Distr-CD-NO spend more time at the server side than Centralised because they using the on demand service.

Nevertheless, this increment is not important compared to response times. In this case peak user requests and network congestion are the most important factors affecting the performance of download operations.

\section{Degraded Mode and Redundancy Costs}

In this section the performance of Upload/Download operations is analysed when Phoenix recovers files that have been allocated in a faulty web server.

In order to compare our results by using a common

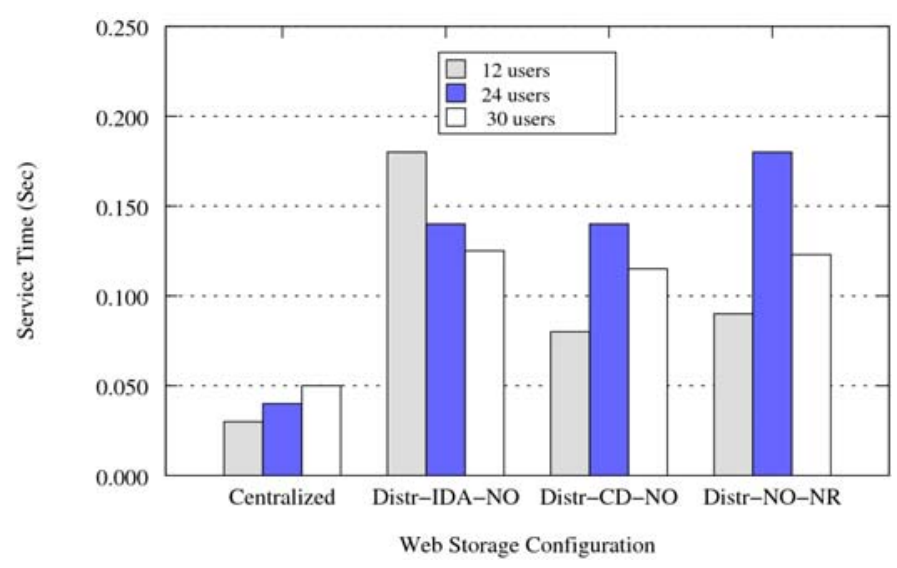

Figure 6. Mean service times of downloading files.

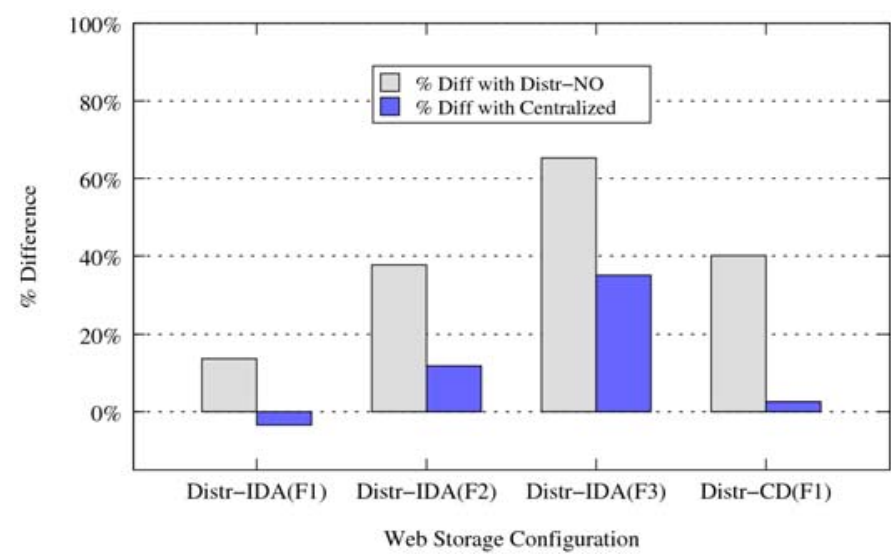

Figure 7. Response time difference between the configurations in normal operation (NO) and degraded mode.

reference, we used Centralised and Distr-NR-NO configurations.

Fig. 7 shows the difference of response times between the configurations working in normal operation (NO) and same configurations working in degraded mode. Only web storage configurations that do not lose data are included in this graph.

As we can see, compared to Distr-IDA-NO, Distr-IDA(Fi) suffers performance penalisation which increases each time another server crashes.

Fig. 7 also shows the same comparison but with the Centralized configuration where we can observe the same behaviour but where a lesser \% of difference is observed. $\operatorname{Distr-IDA}(F 1)$ and Distr-CD(F1) produce similar response times to those obtained from the Centralized configuration.

As we recall, in Distr-CD a given file and its corresponding replica are allocated to two consecutive WS-nodes. In this cyclic order, Distr-CD is only capable of withstanding one single WS-node failure. Distr-IDA supports three failed servers and is still retrieving all the files to the users

Distr-CD produces $100 \%$ capacity overhead even when it is using on demand services. Distr-IDA produces $67.4 \%$ and $167.4 \%$ when configured as a hybrid by using the on demand 
service.

As can be seen, both Distr-CD and Distr-IDA can be implemented at web sites because the load distribution within WS-Cell compensates for the redundancy production and distribution. In addition, when server failures are detected, even the recovery procedures could be also compensated for by the workload distribution.

In Fig. 7 we can also see that Distr-IDA-NO produces similar response times to those values from Distr-NR-NO because both systems provide an on demand service.

\section{CONCLUSIONS}

This paper has presented, in the form of a case study, the design and implementation of a prototype called Phoenix, which is a cost-effective fault-tolerant web storage service.

In our performance assessment we found that the servers' cyclic appointing, the re-utilisation of web metadata and the decoupling between data and metadata turn out to be fundamental design decisions that compensate for the more demanding operations, mainly related to data accommodation and retrieval. Further information on the Phoenix prototype as well as a demo can be seen in [3].

\section{FUTURE WORK}

We are currently extending the capabilities of our prototype. First, we want to offer new redundancy strategies, as well as concurrent access to files. Also, it would be very convenient to extend the overall storage capacities of a cell by adding new web servers on-line, i.e. without the working system coming to a halt. In the near future we are planning the construction of a WSM-cell federation offering several levels of availability and integrity. Finally, we are working on security, compression and encryption approaches as well as semantic search engines. Our goal is to develop a corporative web storage service able to deliver documents based on content.

\section{ACKNOWLEDGMENT}

The authors would like to thank the reviewers for their valuable comments.

\section{REFERENCES}

[1] K. G. Coffman and A. M. Odlyzko, "Internet growth: Is there a "Moore's law” for data traffic?” Handbook of massive data sets, MA, USA: Kulemer Academic Publishers Norwell, 2002, pp. 47-93.

[2] P. Lyman and H. R. Varian, “How much information 2003?” [Online]. Available at: http://www2.sims.berkeley.edu/research/projects/howmuch-info/, Accessed:08/07/2011.

[3] P. N. Yianilos and S. Sobti, “The evolving field of distributed storage," IEEE Internet Computing, vol. 5, no. 5, pp. 35-39, 2001.

[4] J. Kubiatowicz, D. Bindel, Y. Chen, S. Czerwinski, P. Eaton, et al., "OceanStore: An architecture for global-scale persistent storage," SIGPLAN Not., vol. 35, no. 11, pp. 190-201, 2000.

[5] A. Rowstron and P. Druschel, "Storage management and caching in PAST, a large-scale, persistent peer-to-peer storage utility,” Proceedings of the eighteenth ACM Symposium on Operating Systems Principles (SOSP '01), New York, USA, pp. 188-201, 2001.

[6] A. Adya, W. J. Bolosky, M. Castro, G. Cermak, R. Chaiken, et al., "Farsite: federated, available, and reliable storage for an incompletely trusted environment," Proceedings of the 5th Symposium on Operating Systems Design and Implementation (OSDI '02), New York, USA, pp. 1-14, 2002.

[7] H. Weatherspoon and J. Kubiatowicz, "Erasure coding vs. replication: A quantitative comparison,” In P. Druschel, M. F. Kaashoek, and A. I. T.
Rowstron (eds.) Revised Papers from the First International Workshop on Peer-to-Peer Systems (IPTPS '01), London, UK: Springer-Verlag, pp. 328-338, 2002.

[8] Y. Chen, J. Edler, A. Goldberg, A. Gottlieb, S. Sobti, and P. Yianilos, "A prototype implementation of archival inter-memory," Proceedings of the 4th ACM conference on Digital libraries (DL '99), New York, USA, pp. 28-37, 1999.

[9] J. L. Gonzalez and T. Cortes, "Distributing orthogonal redundancy on adaptive disk arrays,” Lecture Notes in Computer Science (OTM 2008), pp. 914-931, 2008.

[10] R. Rodrigues and B. Liskov, "High availability in DHTs: Erasure coding vs. replication,” Proc. 4th International Workshop Peer-to-Peer Systems (IPTPS 05), pp. 226-239, 2005.

[11] T. J. Bittman and L. Leong, "Virtual machines will slow in the enterprise, Grow in the Cloud," Gartner RAS Core Research Note G00210732, 4 March 2011.

[12] R. Yellin, "The data storage evolution: Has disk capacity outgrown its usefulness?” Terada Magazine, 2006.

[13] Uploading.com, [Online]. Available at: http://uploading.com, Accessed: $.08 / 07 / 2011$

[14] Private secure online storage, [Online] Available at: http://www.swissdisk.com, Accessed: 08/07/2011.

[15] Amazon simple storage service (Amazon S3), [Online] Available at: http://aws.amazon.com/s3, Accessed: 08/07/2011 .

[16] A. J. Kevin D. Bowers and A. Oprea, "Hail: A high-availability and integrity layer for cloud storage," Proceedings of the 16th ACM conference on Computer and communications security, pp. 187-198, November 2009, doi:10.1145/1653662.1653686.

[17] E. Chai and M. Uehara, "Online web storage using virtual large-scale disks," Proceedings of Complex, Intelligent and Software Intensive Systems, pp. 512-517, 2009.

[18] E. Chai and M. Uehara, "Virtual large-scale disk system for pc-room," LNCS 4658, Network-Based Information Systems, pp. 476-485, 2007.

[19] M. Quezada-Naquid, R. Marcelín-Jiménez, and M. Lopez-Guerrero, "Fault tolerance and load balance tradeoff in a distributed storage system,” Computación y Sistemas (ISSN 1405-5546), vol. 14, no. 2, 2010.

[20] H. I. Hsiao and D. J. DeWitt, "Chained declustering: A new availability strategy for multiprocessor database machines,” Proceedings of the Sixth International Conference on Data Engineering, pp. 456-465, 5-9 Feb 1990.

[21] M. O. Rabin, "Efficient dispersal of information for security, load balancing, and fault tolerance," Journal of the ACM, vol. 36, no. 2, pp. 335-348, 1989.

[22] R. Marcelin-Jimenez, S. Rajsbaum, and B. Stevens, "Cyclic Storage for Fault-Tolerant Distributed Executions,” IEEE Trans. Parallel Distrib. Syst., vol. 17, no. 9, pp. 1028-1036, 2006.

[23] Phoenix prototype, [Online] Available at: www.adaptivez.org.mx, Accessed: 08/07/2011. 
This page is intentionally left blank 